\title{
Copula-based Measurement of Dependence Between Dimensions of Well-being
}

\author{
Koen Decancq*
}

December 2, 2009

\begin{abstract}
Well-being consists of many dimensions such as income, health and education. A society exhibits greater dependence between its dimensions of well-being when the positions of the individuals in the different dimensions are more aligned or correlated. Differences in dependence may lead to very different societies, even when the dimension-wise distributions are identical. I propose to use a copula-based framework to order societies with respect to their dependence. A class of measures of dependence is derived to which the multidimensional rank correlation coefficient belongs. I illustrate the usefulness of the approach by showing that Russian dependence between three dimensions of well-being has increased significantly between 1995 and 2003. Unfortunately, the aspect of dependence is missed by all composite well-being measures based on dimension-specific summary statistics such as the popular Human Development Index (HDI).
\end{abstract}

Keywords: copula, complex inequality, concordance, HDI, multidimensional inequality, Russia, well-being.

JEL Classification: D31, D63, I31, O50.

${ }^{*}$ Koen Decancq: Center for Economic Studies, Katholieke Universiteit Leuven, Naamsestraat 69, B3000 Leuven, Belgium. E-mail: koen.decancq@econ.kuleuven.be.

This is a revised version of a chapter of my Ph.D. dissertation. I thank André Decoster, James E. Foster, Luc Lauwers, Erwin Ooghe, Casey Quinn, Erik Schokkaert, Suman Seth, Tom Van Ourti, John A. Weymark and participants at seminars in Leuven, Berlin, Montreal, Verona, Oxford, and Louvain-LaNeuve for very helpful comments and suggestions to this or an ealier version of the paper. All comments are warmly welcomed. Remaining errors are mine.

I also thank the Russia Longitudinal Monitoring Survey Phase 2, funded by the USAID and NIH (R01HD38700), Higher School of Economics and Pension Fund of Russia, and provided by the Carolina Population Center and Russian Institute of Sociology for making these data available. 
"Consider institutional schemes under which half the population are poor and half have no access to higher education. We may plausibly judge such an order to be more unjust when the two groups coincide than when they are disjoint (so that no one bears both hardships)."

(Pogge 2002)

\section{Introduction}

Individual well-being is multidimensional. When individuals consider how well-off they are, they typically take into account their outcomes in many dimensions of life, such as their income, health or schooling to name a few. Individuals implicitly aggregate and trade-off all these outcomes to obtain an overall judgment of their well-being.

If we want to take this multidimensionality into account when considering how well-off a society is as a whole, we face a double aggregation problem: one across the dimensions of well-being and one across the individuals of the society. To do so, two approaches exist in the literature (Dutta, Pattanaik, and Xu 2003). One approach is to first aggregate across the different dimensions for every individual to obtain a measure of her well-being, and then in a second step to aggregate the resulting well-being measures across the individuals. Such an approach is in line with standard welfare economic theory, but poses heavy requirements on the data, since information on many dimensions has to be collected for the same (representative) individuals. An alternative approach first calculates summary statistics for every separate dimension, which are afterwards aggregated across the different dimensions. This approach has the advantage that it allows the dimension-specific summary statistics to be based on different data sources. However, this flexibility comes at a price: an important aspect of the information gets lost, namely the correlation or dependence between the positions of the individuals across the different dimensions.

Compare, for instance, the following two societies. In the first society, there is one individual who is top ranked in all dimensions of well-being, another individual second ranked in all dimensions and so on. This society, reminiscent of a feudal or castesystem, is arguably less equitable than another one with exactly the same distributional profile in each dimension, but where some individuals are performing relatively well on some dimensions and other individuals on other dimensions. Despite the difference in the correlation or dependence between the dimensions, both societies are judged to be equally well-off by all well-being measures that summarize first the information in every dimension, and then aggregate across dimensions. The insensitivity to dependence is 
an important drawback of all well-being measures based on summary statistics of their dimensions. The most prominent example of such a measure is the popular Human Development Index (HDI), which includes the society's per capita material standard of living, its average life expectancy and its average educational outcomes.

The study of dependence is not only interesting to quantify the blind spot of multidimensional well-being measures such as the HDI, the dependence between the dimensions of well-being per se plays a central role in the pluralistic theory of distributive justice. The political philosopher Michael Walzer (1983) distinguishes in his theory between simple and complex inequality. Whereas simple equality requires an egalitarian distribution in some central dimensions of well-being, complex equality is achieved when the dimensions are autonomous or independent. In his words, "no individual's standing with regard to one good can be undercut by his standing with regard to some other good" (Walzer 1983). A society with maximal complex equality is one in which the dimensions are completely independent of each other. ${ }^{1}$ Walzer argues that rich individuals, for instance, should be prohibited to use their high income to buy votes, to obtain better health care or educational outcome and so on. A caste system, on the other hand, can be seen as an example of a society with maximal complex inequality. Yet, when it comes to ordering societies in between these extremes with respect to their complex inequality or to measuring complex equality, the theory offers limited guidance. Swift (1995) states “... one must at least say that people are more [complex] equal if those who score high with respect to some goods score low with respect to others than if the same people score high (and low) on all" and Miller (1995) suggests that there is more complex inequality in a society when there are more individuals who outrank each other in all dimensions of well-being.

This essay proposes an applicable framework to order and quantify the dependence of societies. In this framework, the copula function will play a central role. The copula allows one to disentangle the information on the dependence structure from the information on the dimension-wise distributional profiles in each dimension of well-being. In this way, the dependence of societies with different dimension-wise distributional profiles can be compared. I study the properties of the copula-based concordance dependence relation and characterize axiomatically a family of dependence measures which is consistent with it.

Contrary to their popularity in actuarial sciences to describe and model multidimensional

\footnotetext{
1 The notion of complex equality has been criticized for being a very weak egalitarian concept. For instance, Arneson (1995) argues that the ideal of complex equality can coexist with a large spread between the top and bottom in the distributional profile of every separate dimension. Walzer admits that there may exist inequalities in each dimension, but argues that given the multidimensionality of well-being individuals will perform relatively well in at least some dimensions, so that somehow the inequalities will be compensated across dimensions.
} 
risks, copula-based approaches have been applied rarely in welfare economics. Dardanoni and Lambert (2001) use a copula-based framework to measure the extent of re-ranking taking place through taxation. The rank-correlation approach used by Fournier (2001) to analyze the correlation between the incomes of spouses is also implicitly copula-based. Bonhomme and Robin (2006) estimate various parametric copulas to describe the individual earnings trajectories and income mobility in France. Quinn (2007, 2009) analyses the correlation between health and income by a copula-based measure. All of these applications are limited to the two-dimensional setting. The multidimensional generalization proposed in this essay moves beyond the particular two-dimensional setting.

The usefulness of the proposed copula-based partial dependence relations and a consistent dependence measure is illustrated by addressing the question whether the dependence between the dimensions of well-being has increased in the Russian Federation between 1995 and 2003. I use data from the Russian Longitudinal Monitoring Surveys (RLMS) and focus on the three dimensions of well-being included in the HDI: standard of living, health and schooling. The RLMS is a particularly rich panel data set that follows a wide range of outcomes for the same individuals over a relatively long period and, hence, allows one to quantify dependence between the dimensions in a detailed way. After the collapse of the Soviet Union, Russia underwent a fast and far-reaching transition from a centrally planned economy to a market economy. During this transition, the access to social services such as health care, schooling and housing, which used to be widespread and free, increasingly hinged on the ability to pay for them. ${ }^{2}$ These observations suggest an increased dependence between the Russian dimensions of well-being after the transition. Moreover, the copula-based analysis of dependence in the Russian society after the transition sheds some light on a structural blind spot of the HDI: its insensitivity to dependence between dimensions of well-being.

The essay is structured as follows. In the second section I introduce the copula and some of its basic properties. In section three a partial dependence relation based on dominance of the copula function is defined. Section four derives the family of dependence measures that are consistent with the partial dependence relation, thereby addressing an open question raised by Dardanoni and Lambert (2001, p. 808): "The interesting question, to identify the class of copula-based indices which accord with our [concordance] partial ordering, remains open and is clearly an important one for future research." Prominent members of the resulting class will turn out to be multidimensional generalizations of existing two-dimensional association measures, such as Spearman's rank correlation

\footnotetext{
${ }^{2}$ Blam and Kovalev (2006), Besstremyannaya (2007) and Lokshin and Ravallion (2008) document the recent commercialization in the Russian health care sector and Smolentseva (2007) investigates the increasing educational inequalities due to the partial privatization of the schooling system.
} 
coefficient. Section five illustrates the derived dominance criteria and measures of dependence by testing whether the dependence in Russia has increased between 1995 and 2003. Section six concludes.

\section{The copula}

Let there be $m$ relevant dimensions of well-being, such as material standard of living, health and schooling. Consider a population of individuals $\Omega$. The random vector $X=$ $\left(X_{1}, \ldots, X_{m}\right)$ attaches to every individual in $\Omega$ an $m$-dimensional real well-being vector. Let $F_{X}: \mathbb{R}^{m} \rightarrow[0,1]$ be the joint distribution function of the random vector $X$. Hence, for each $x$ in $\mathbb{R}^{m}$, we have:

$$
F_{X}\left(x_{1}, \ldots, x_{m}\right)=\operatorname{Pr}\left[X_{1} \leq x_{1} \text { and } \ldots \text { and } X_{m} \leq x_{m}\right]
$$

where $\operatorname{Pr}$ is a probability measure on $\Omega$. When there is no confusion about the underlying random vector $X$, the joint distribution function will be simply denoted $F$. The marginal distribution function of dimension $j, F_{j}: \mathbb{R} \rightarrow[0,1]$ gives for every outcome $x_{j}$ the probability that an individual from $\Omega$ has weakly less in dimension $j$ than $x_{j}$,

$$
F_{j}\left(x_{j}\right)=\operatorname{Pr}\left[X_{j} \leq x_{j}\right]
$$

The position $p_{j}=F_{j}\left(x_{j}\right)$ is a real number between 0 and 1 and can be interpreted as the population variant of the rank of the individual having $x_{j}$ in the total distribution of dimension $j$. The position vector $p=\left(p_{1}, \ldots, p_{m}\right)$ of an individual lists her positions in all dimensions of well-being. The individual with a position vector equal to $(1, \ldots, 1)$ is top ranked in all dimensions, whereas the individual with position vector $(0, \ldots, 0)$ is in all dimensions bottom ranked. A position vector is said to be outranked or vector-dominated by another one, if it has a lower or equal position in all dimensions. Similarly, one position vector outranks another one if it has a higher or equal position in all dimensions.

The random variable $P_{j}$ attaches to every individual in $\Omega$ her position in the $j$-th dimension of well-being. The random variable $P_{j}$ is obtained by transforming the original random variable $X_{j}$ by its marginal distribution function, so that $P_{j}=F_{j}\left(X_{j}\right)$. From probability theory, $P_{j}$ is known to follow a standard uniform distribution. The random vector $P$ collects the random variables $P_{j}$ so that $P=\left(F_{1}\left(X_{1}\right), \ldots, F_{m}\left(X_{m}\right)\right)$. The joint distribution function of $P$ is a so-called copula function, denoted $C{ }^{3}$ The copula function will be referred to as $C_{X}$ when it is advantageous to make the identification with the

\footnotetext{
${ }^{3}$ See Schweizer and Sklar (1983), Joe (1997) or Nelsen (2006) for an extensive treatment of the definition and properties of the copula function.
} 
underlying random vector $X$ explicit. Formally, each copula function is defined as a joint distribution function with standard uniform marginal distribution functions.

Definition 1. An m-dimensional copula function $C:[0,1]^{m} \rightarrow[0,1]$ is an m-dimensional distribution function whose support is the $m$-dimensional unit hyperbox $[0,1]^{m}$ and whose one-dimensional marginal distribution functions follow a standard uniform distribution. Hence for all $p=\left(p_{1}, \ldots, p_{m}\right)$ in $[0,1]^{m}$ :

$$
C\left(p_{1}, \ldots, p_{m}\right)=\operatorname{Pr}\left[P_{1} \leq p_{1} \text { and } \ldots \text { and } P_{m} \leq p_{m}\right]
$$

The copula function maps every position vector $p$ to the probability that a realization from random vector $P$ is outranked by that position vector. Similarly, the probability that a realization from random vector $P$ outranks a given position vector $p$, is given by the joint survival function, denoted $\bar{C}:[0,1]^{m} \rightarrow[0,1]$. For all $p$ in $[0,1]^{m}$, we say:

$$
\bar{C}\left(p_{1}, \ldots, p_{m}\right)=\operatorname{Pr}\left[P_{1} \geq p_{1} \text { and } \ldots \text { and } P_{m} \geq p_{m}\right]
$$

Every copula function is a joint distribution function defined on the unit hyperbox $[0,1]^{m}$. Conversely, Sklar (1959) has shown that each joint distribution function $F_{X}$ can be written as a function of its $m$ one-dimensional marginal distribution functions $F_{j}$ and its copula function $C_{X}$.

Theorem 1 (Sklar, 1959). Let $X$ be a random vector with joint distribution function $F_{X}$ and marginal distribution functions $F_{1}, \ldots, F_{m}$. Then there exists a copula function $C_{X}$ such that for all $x$ in $\mathbb{R}^{m}$ :

$$
F_{X}\left(x_{1}, \ldots, x_{m}\right)=C_{X}\left(F_{1}\left(x_{1}\right), \ldots, F_{m}\left(x_{m}\right)\right) .
$$

Moreover, $C_{X}$ is uniquely determined on Range $\left(F_{1}\right) \times \cdots \times$ Range $\left(F_{m}\right)$.

The copula is the function that maps the marginal distribution functions on their joint distribution function so that the joint distribution function $F_{X}$ (expression (1)) can be retrieved from the marginal distributions $F_{1}, \ldots, F_{m}$ (expression $(2)$ ) and the copula function $C_{X}$ (expression (3)). Conversely, Sklar's result permits one to decompose the $m$-dimensional joint distribution function into the marginal distribution functions and the copula function. The marginal distribution functions represent the distribution of the outcomes in the different dimensions of well-being. The copula function, on the other hand, captures the dependence between the dimensions. After all, the more the positions coincide across dimensions, the larger the probability that a realization from $P$ is outranked by a given position vector $p$ and, hence, the larger the value of the copula 
function. In this essay, the copula function $C_{X}$ of the random vector $X$ will be the central building block in the analysis of the dependence in the society represented by $X$.

An intuitive example of a copula function is the independent copula $C_{\perp}(p)=p_{1} \times \cdots \times p_{m}$. Using $p_{j}=F_{j}\left(x_{j}\right)$ and Sklar's theorem, the independence joint distribution function can indeed be retrieved as the product of its marginal distribution functions. In the literature many other parametric families of copulas have been studied, see Joe (1997) and Nelsen (2006) for an overview.

The notion of dependence used in this essay is based on the alignments of the positions in the different dimensions, rather than on the levels of the outcomes. Schweizer and Wolff (1981) note: “... it is precisely the copula which captures those properties of the joint distribution which are invariant under strictly increasing transformations". Let $\Phi(X)=\left(\phi_{1}\left(X_{1}\right), \ldots, \phi_{m}\left(X_{m}\right)\right)$ denote the transformed random vector obtained by applying dimension-specific strictly increasing continuous transformation functions $\phi_{1}, \ldots, \phi_{m}$ to the respective random variables. A transformation of a dimension changes the levels of the outcomes of the individuals without changing their positions. Since a copula is a function of the position vectors only, it is invariant to any strictly increasing transformation of the dimensions of its underlying random vector $X$. Formally, Schweizer and Wolff (1981) prove the following result.

Theorem 2 (Schweizer and Wolff, 1981). Let X be a random vector with copula function $C_{X}$ and let the transformed random vector $\Phi(X)$ be obtained by applying strictly increasing transformation functions $\phi_{1}, \ldots, \phi_{m}$ to its random variables respectively, then $C_{X}=C_{\Phi(X)}$.

The result by Schweizer and Wolff (1981) shows the usefulness and limitations of a copula-based analysis of dependence. The copula function focusses on the dependence or correlation between the positions of the individuals in the different dimensions, irrespective of the exact levels of the outcomes that correspond to these positions. A copula-based analysis of dependence can be based on ordinal indicators in a way that is robust to the exact scaling procedure. On the other hand, a copula-based analysis cannot take any information into account about the exact levels of the outcomes. A copula-based approach cannot replace a fully-fledged multidimensional inequality analysis. ${ }^{4}$ However, the result by Sklar (1959) suggests that there is room for a complementary approach, in which the standard dimension-by-dimension analysis focussing on the distribution of the outcome levels within every dimension is complemented with a copula-based analysis focussing on the ordinal information about the dependence between the dimensions.

\footnotetext{
${ }^{4}$ Maasoumi (1999) and Weymark (2006) provide excellent overviews of the literature on multidimensional inequality measurement.
} 
Each copula function is bounded by its so-called Fréchet-Hoeffding bounds, reflecting the cases of minimal and maximal dependence, which are defined by $C_{-}(p)=\max \left(p_{1}+\right.$ $\left.\ldots+p_{m}-m+1,0\right)$ and $C_{+}=\min \left(p_{1}, \ldots, p_{m}\right)$ respectively.

Theorem 3 (Fréchet-Hoeffding). If $C$ is a copula function, then for all $p$ in $[0,1]^{m}$ :

$$
C_{-}(p) \leq C(p) \leq C_{+}(p)
$$

The copula function reflecting maximal dependence, the so-called comonotonic copula $C_{+}(p)$, represents the dependence structure in which the outcomes in all dimensions are ordered in the same way. In other words, $C_{+}$is the copula function of the random vector $X_{+}$in which every random variable $X_{j}$ is an increasing function of every other random variable $X_{k}$. Consequently, for any given position vector $p$, all the other position vectors are either outranking or outranked by $p$. A joint distribution function with a comonotonic copula describes a society in which one individual is top-ranked in all dimensions, another is second-ranked and so forth. This society is maximally unequal in the complex sense defined by Walzer (1983). Dardanoni (1996) refers to a distribution with a comonotonic copula when he talks about a distribution after an unfair rearrangement.

Things are a little bit more intricate when it comes to defining the lower bound. The function $C_{-}$describes the dependence structure of the so-called countermonotonic random vector $X_{-}$. For $m \geq 3$, the function $C_{-}$is formally not a distribution function and, hence, can not be a copula function (Nelsen 2006, p.47). Nevertheless, in cases of countermonotonicity, high ranks in some dimensions come with low ranks in others and none of the position vectors $p$ outranks or is outranked by any other position vector. In other words, there is no pair of individuals such that the well-being vector of one individual vector-dominates the one of the other, so that a countermonotonic society satisfies the no-dominance equity axiom used in fair allocation theory (Moulin and Thomson 1997, Fleurbaey and Trannoy 2003).

Walzer refers to the case in which the dimensions are autonomous or independent as the ethical benchmark of a maximally complex equal society. Independence is captured by the independent copula $C_{\perp}$. If all the dimensions are independent, the position of an individual in one dimension does not depend on her position in any other dimension. ${ }^{5}$

\footnotetext{
5 Note that independence of the dimensions does not exclude that some individuals may outrank others. Hooghe (1999) refers to Alcibiades as an ancient example of such an exceptionally gifted individual who outranks all others individuals in many dimensions. The more relevant dimensions there are, the smaller the chance that outranking occurs in a society with independent dimensions.
} 


\section{Orderings of dependence}

The copula function $C_{X}$ of a random vector $X$ contains all information about the dependence structure. We can therefore order random vectors by comparison of their copula functions. Let $\succsim$ denote a dependence relation on the set of random vectors $X$, which should be interpreted as meaning 'is weakly more dependent than'. The corresponding strict and equivalence relations are denoted $\succ$ and $\sim$. In the rest of this section I will survey three specific dependence relations that make use of the copula function, the first of which is the lower orthant dependence relation. ${ }^{6}$

Definition 2. Let $X$ and $Y$ be two random vectors with copula functions $C_{X}$ and $C_{Y}$ respectively. Then $Y$ is said to be weakly more dependent than $X$ according to the lower orthant dependence relation, denoted $Y \succsim_{L O} X$, if for all $p$ in $[0,1]^{m}$ :

$$
C_{Y}(p) \geq C_{X}(p)
$$

Figure (1) illustrates this condition in the two-dimensional case. On both axes of the figure, the positions of the individuals are shown. Individuals that are top ranked in both dimensions will be in the top-right-hand corner of $[0,1]^{2}$. A random vector with comonotonic copula $C_{+}$will have all individuals on the diagonal. In the case of independence they are evenly scattered on $[0,1]^{2}$. Condition (4) implies that for each position vector $p$ in $[0,1]^{2}$, the proportion of the society in its lower orthant (the light shaded area on the figure), that is the proportion of individuals who are outranked by $p$, is not smaller in the society with random vector $Y$ than $X$. This condition has to hold for all position vectors, hence the lower orthant dependence relation is a partial dependence relation.

Two random vectors $X$ and $Y$ are equivalent in the lower orthant dependence relation, if they have the same copula function or, equivalently, if in every dimension $j$ the random variable $Y_{j}$ can be obtained from $X_{j}$ by a strictly increasing transformation function.

The lower orthant dependence relation is a special case of the multidimensional firstorder stochastic dominance ordering studied by Hadar and Russel (1974), Epstein and Tanny (1980) and Atkinson and Bourguignon (1982) amongst others. The multidimensional joint distribution function $F_{Y}$ is said to stochastically dominate $F_{X}$ in the first order if $F_{Y}(x) \geq F_{X}(x)$ holds for all $x$ in $\mathbb{R}^{m}$, where $F_{Y}$ and $F_{X}$ are two joint distribution functions with the same marginal distribution functions. Indeed, any two copula

${ }^{6}$ The idea of ordering random vectors with respect to their dependence based on dominance of their underlying copulas dates back to the work of Scarsini (1984). For a recent survey on dependence relations, see Colangelo, Scarsini and Shaked (2006). 


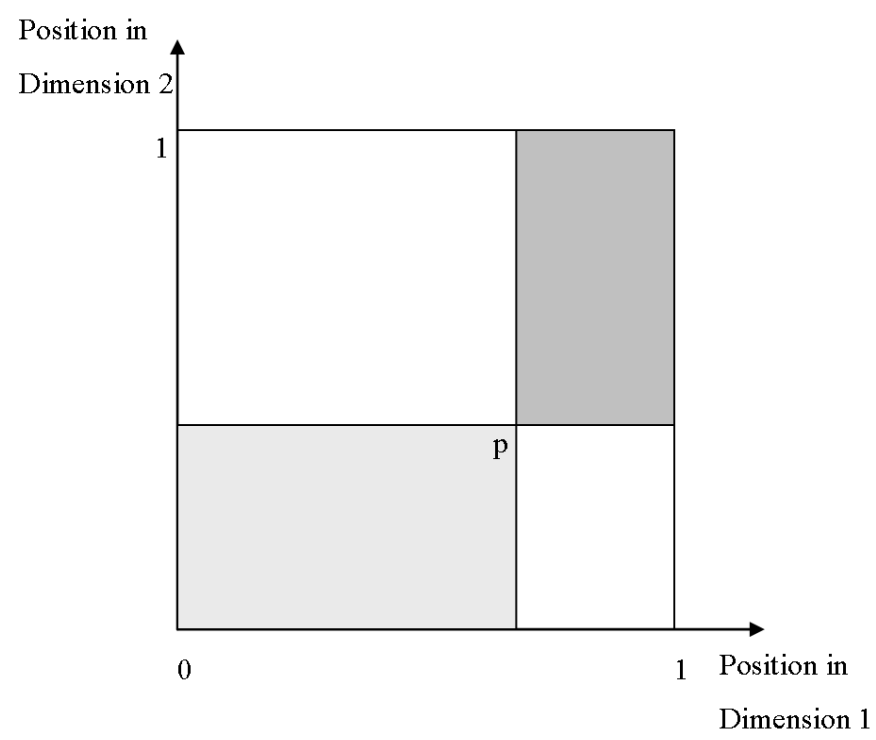

Figure 1: Lower and upper orthants of $p$ in $I^{2}$.

functions are joint distribution functions with the same (standard uniform) marginal distribution functions. However, comparing the underlying copula functions instead of the joint distribution functions themselves has the advantage that it also allows one to compare the extent of dependence in joint distribution functions with different marginal distributions.

The mirror image of the lower orthant dependence relation is the upper orthant dependence relation. It is based on dominance of the joint survival function and can be defined analogously.

Definition 3. Let $X$ and $Y$ be two random vectors, with joint survival functions $\bar{C}_{X}$ and $\bar{C}_{Y}$ respectively. Then $Y$ is said to be weakly more dependent than $X$ according to the upper orthant dependence relation, denoted $Y \succsim_{U O} X$, if for all $p$ in $[0,1]^{m}$ :

$$
\bar{C}_{Y}(p) \geq \bar{C}_{X}(p) \text {. }
$$

Condition (5) requires that for each position vector $p$, the probability that a realization of $Y$ outranks $p$ is not smaller than the one for $X$. In the two-dimensional setting depicted in Figure 1, the upper orthant of $p$ is the dark shaded area. In a two-dimensional framework, the lower and upper orthant dependence relation are equivalent, ${ }^{7}$ but once

\footnotetext{
${ }^{7}$ This follows from the fact that in the two-dimensional setting:

$$
\bar{C}\left(p_{1}, p_{2}\right)=1-C\left(1, p_{2}\right)-C\left(p_{1}, 1\right)+C\left(p_{1}, p_{2}\right)
$$$$
=1-p_{2}-p_{1}+C\left(p_{1}, p_{2}\right) \text {. }
$$ 
there are more than two dimensions, this is no longer the case.

A third dependence relation combines both orthant dependence relations. This dependence relation is the so-called concordance dependence relation. ${ }^{8}$

Definition 4. Let $X$ and $Y$ be two random vectors, with copula functions $C_{X}$ and $C_{Y}$ and joint survival functions $\bar{C}_{X}$ and $\bar{C}_{Y}$ respectively. Then $Y$ is said to be weakly more dependent than $X$ according to the concordance dependence relation, denoted $Y \succsim_{C} X$, if for all $p$ in $[0,1]^{m}$ :

$$
C_{Y}(p) \geq C_{X}(p) \text { and } \bar{C}_{Y}(p) \geq \bar{C}_{X}(p) .
$$

It is easily checked that the concordance dependence relation (as well as the lower and upper orthant dependence relations) is reflexive, transitive and incomplete, hence formally an incomplete pre-ordering. In the two-dimensional case, all three dependence relations are equivalent. In general, $Y \succsim_{C} X$ implies $Y \succsim_{U O} X$ and $Y \succsim_{L O} X$, and if both $Y \succsim_{U O} X$ and $Y \succsim_{L O} X$ hold, $Y \succsim_{C} X$ is implied. The minimal element of all three dependence relations is the random vector $X_{-}$with a countermonotonic dependence structure and the maximal element is the random vector $X_{+}$which has a comonotonic copula.

\section{Measures of dependence}

The dependence relations defined in the preceding sections are incomplete dependence relations. Some pairs of random vectors can be ranked, but for some other pairs the dependence relation is 'indecisive'. Although the indecisiveness might be informative in its own right, in many situations one may prefer a complete dependence relation that can rank all random vectors with respect to their dependence.

For notational clarity, I will denote a complete binary relation on the set of random vectors by $\succsim^{*}$ with the corresponding strict and equivalence relations $\succ^{*}$ and $\sim^{*}$, respectively. A measure of dependence $D$ is a real-valued order-preserving function of $\succsim^{*}$ that satisfies $Y \succsim^{*} X \Rightarrow D(Y) \geq D(X)$. I follow an axiomatic approach to derive the class of dependence measures that satisfies a minimal set of logically independent axioms on

\footnotetext{
${ }^{8}$ See Joe $(1990,1997)$ or Nelsen $(2006)$ on the concordance ordering. Joe (1990) uses a slightly more general definition based on two joint distribution functions with the same margins, of which the copula with its standard uniform margins is but one example. Decancq (2009) analyses the rearrangements leading to a more dependent society according to the concordance dependence relation.
} 
${ }^{*}{ }^{9}$ Formally, the problem of ordering random vectors resembles the problem of ordering lotteries and, hence, the first three axioms can be borrowed from the literature on expected utility representations. The fourth axiom adds the notion of dependence.

The first axiom requires $\succsim^{*}$ to be a complete and transitive binary relation so that indecisiveness or cycles are excluded.

Weak Ordering (ORD). The binary relation $*^{*}$ is transitive and complete on the set of random vectors.

The following two axioms involve the properties of linear convex combinations of random vectors. Formally, we define a linear convex combination of random vectors $X$ and $Y$ by $\lambda X+(1-\lambda) Y$ for every $\lambda$ in $[0,1]$. A linear convex combination of two random vectors $X$ and $Y$ is again a random vector and in the setting of this essay, it can be interpreted as a unification of two societies with their own dependence structure where $\lambda$ reflects the population share of the first society in the unified total society.

The second axioms adds a flavor of continuity and requires that if $X \succsim^{*} Y \succsim^{*} Z$ holds, then there exist at least one linear convex combination of $X$ and $Z$ that is ordered on either side of $Y$.

Continuity (CONT). For all random vectors $X, Y$ and $Z$, if $X \succsim^{*} Y \succsim^{*} Z$, then there exist an $\alpha$ and $\beta$ in $(0,1)$, such that $\alpha X+(1-\alpha) Z \succsim^{*} Y$ and $Y \succsim^{*} \beta X+(1-\beta) Z$.

The third axiom imposes that $\succsim^{*}$ is maintained after a linear convex combination is taken with a common random vector $Z$.

Independence (IND). For all random vectors $X, Y$ and $Z$, if $X \succsim^{*} Y$ and $\lambda$ in $(0,1)$, then $\lambda X+(1-\lambda) Z \succsim^{*} \lambda Y+(1-\lambda) Z$.

These three axioms together impose a general (additively separable) structure on $\succsim^{*}$, but so far we have not imposed any sensitivity to dependence to $\succsim^{*}$. The next axiom ensures that the ordering of two random vectors implied by $\succsim^{*}$ accords with the ordering of two random vectors by $\succsim_{C}$ which restricts the attention to copula-based measures. ${ }^{10}$

\footnotetext{
${ }^{9}$ The aim of this axiomatic approach is different from the axiomatic approach followed in the statistical literature by Scarsini (1984), Dolati and Úbeda-Flores (2006) and Taylor (2007), who check a series of statistical properties for a given class of measures.

10 By the theorem of Schweizer and Wolf (1981), all copula-based measures are invariant to strictly increasing continuous transformations of the dimensions. The standard (Pearson) correlation coefficient satisfies a much weaker invariance property, specifically it is invariant to strictly increasing linear transformations of the dimensions. On the potential pitfalls of using a non-copula-based measure such as the standard correlation coefficient as measure of dependence, see Embrechts et al. (2000).
} 
Consistency with the concordance dependence relation (CONC). For all random vectors $X$ and $Y$, if $Y \succsim_{C} X$, then $Y \succsim^{*} X$.

The following Proposition derives the class of order preserving dependence measures $D$ which satisfies these four axioms. To state this Proposition I make use of a realvalued function $S$ whose even cross derivatives are all non-negative. In other words, $\partial^{k} S(p) / \partial p_{j_{1}} \ldots \partial p_{j_{k}} \geq 0(k=2,4, \ldots)$ holds for all sets of an even number of dimensions. ${ }^{11}$

Proposition. If the binary relation $\succsim^{*}$ on the set of random vectors satisfies ORD, $C O N T, I N D$ and $C O N C$, and if the real-valued function $S$ has non-negative even crossderivatives, then $Y \succsim^{*} X$ implies that:

$$
D(Y)=\int_{[0,1]^{m}} S(p) d C_{Y}(p) \geq \int_{[0,1]^{m}} S(p) d C_{X}(p)=D(X) .
$$

Proof. See Appendix.

Each function $S$ defines a different measure of dependence or complex inequality that is consistent with the concordance dependence relation.

In the statistical literature on the measurement of dependence a particular subclass of functions $S$ with non-negative even cross-derivatives has been studied, that is the class in which $S$ can be expressed as a sum of a copula function and joint survival function of the same reference random vector $Z .^{12}$ In this case, we say that:

$$
D_{Z}(X)=\int_{[0,1]^{m}}\left[C_{Z}(p)+\bar{C}_{Z}(p)\right] d C_{X}(p) .
$$

Let the marginal distribution function of random variable $X_{j}$ be denoted by $F_{j}$ and the marginal distribution function of random variable $Z_{j}$ be denoted by $G_{j}$. By the definition of the copula function and joint survival function, it follows that:

$$
\begin{aligned}
D_{Z}(X)= & \operatorname{Pr}\left[G_{1}\left(Z_{1}\right) \leq F_{1}\left(X_{1}\right) \text { and } \ldots \text { and } G_{m}\left(Z_{m}\right) \leq F_{m}\left(X_{m}\right)\right] \\
& +\operatorname{Pr}\left[G_{1}\left(Z_{1}\right) \geq F_{1}\left(X_{1}\right) \text { and } \ldots \text { and } G_{m}\left(Z_{m}\right) \geq F_{m}\left(X_{m}\right)\right] .
\end{aligned}
$$

The dependence measure $D_{Z}(X)$ gives the probability that the realization of an individual from $\Omega$ according to the random vector $X$ outranks or is outranked by the realization

\footnotetext{
11 This proposition is a specific case of a result by Decancq (2009).

12 See for instance, Nelsen (1991, 2002), Dolati and Úbeda-Flores (2006), Schmid and Schmidt (2006, 2007) Behboodian, Dolati and Úbeda-Flores (2007) and Taylor (2007). It can easily be checked that the sum of a copula and joint survival function satisfies the restriction imposed by the proposition.
} 
according to $Z$. Nelsen $(1991,2002)$ calls this "the probability of concordance" and uses it as building block for the derivation of various measures of dependence.

It is convenient for the purpose of measuring complex inequality to further require that the dependence of the benchmarks $X_{\perp}$ (autonomous or independent dimensions) and $X_{+}$ (comonotonic or maximally correlated dimensions) is normalized to 0 and 1 respectively. To achieve that, a linear normalization of $D_{Z}(X)$ is employed that leads to dependence index $\widetilde{D}_{Z}(X)$ :

$$
\widetilde{D}_{Z}(X)=\frac{D_{Z}(X)-D_{Z}\left(X_{\perp}\right)}{D_{Z}\left(X_{+}\right)-D_{Z}\left(X_{\perp}\right)}
$$

The normalized dependence index $\widetilde{D}_{Z}(X)$ can be interpreted as the relative position between the benchmarks of independence $X_{\perp}$ and maximal dependence $X_{+}$.

An interesting choice for the reference random vector $Z$ is the independent random vector $Y_{\perp} \cdot{ }^{13}$ After some algebraic manipulations the resulting dependence index $\widetilde{D}_{\perp}(X)$ equals:

$$
\widetilde{D}_{\perp}(X)=\frac{(m+1)\left[2^{m-1} \int_{[0,1]^{m}}\left[C_{\perp}(p)+\bar{C}_{\perp}(p)\right] d C_{X}(p)-1\right]}{2^{m}-(m+1)},
$$

which is a multidimensional generalization of the two-dimensional Spearman rank correlation coefficient $\rho .^{14}$ The dependence index $\widetilde{D}_{\perp}(X)$ has an interesting interpretation in terms of complex inequality. It equals the normalized probability that a randomly drawn individual from a given society $X$ outranks or is outranked by a randomly drawn individual from a reference society which is Walzer's ideal society $Z_{\perp}$ with independent dimensions. The more dependence or correlation there is in $X$ the higher this normalized probability.

\section{The Evolution of the Dependence between Dimensions of Well-being in Russia.}

In this section, the empirical usefulness of the multidimensional concordance dependence relation and the copula-based dependence measures is illustrated based on data from the Russian Longitudinal Monitoring Survey (RLMS) between 1995 and 2003. ${ }^{15}$ I will

\footnotetext{
${ }^{13}$ Alternatively, if $Y$ is set to be equal to the random vector $X$ itself, the dependence index $\widetilde{D}_{X}(X)$ is a multidimensional generalization of Kendall's $\tau$ and belongs to the family of generalizations of Kendall's $\tau$ proposed by Joe (1990) (Nelsen 1991, Nelsen 2002). It equals the normalized probability that two randomly drawn individuals from the society $X$ outrank each other.

14 The dependence index $\widetilde{D}_{\perp}(X)$ is the mean of two multidimensional rank correlation coefficients $\rho$ discussed in Joe (1990) and has been studied by Nelsen (1991, 2002), Dolati and Úbeda-Flores (2006), Schmid and Schmidt (2006, 2007) and Taylor (2007).

15 The RLMS consists of a series of nearly annual, nationally representative surveys designed to monitor the effects of Russian reforms on the health and economic welfare of households and individuals in the Russian Federation. I use rounds VI-XII of the survey which took place in 1995, 1996, 1998, 2000, 2001, 2002 and 2003.
} 


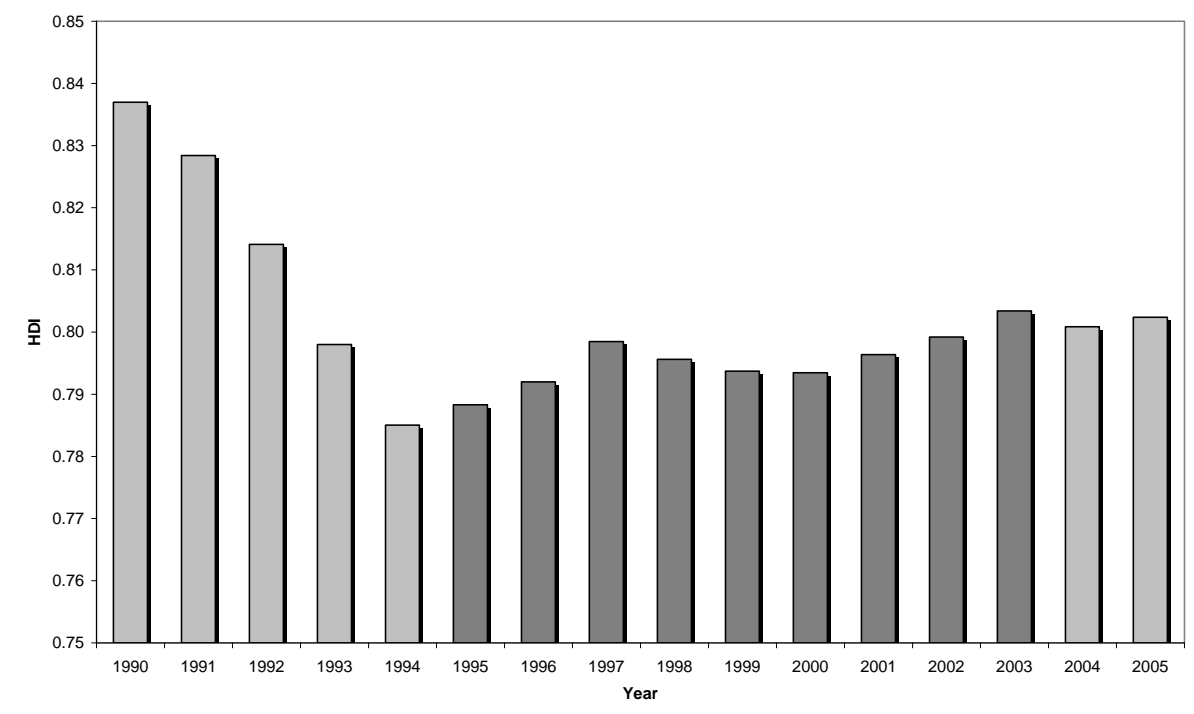

Figure 2: Human Development Index of the Russian federation (Source: author's calculations based on data from the World Development Indicators, 2008).

test the hypothesis of increased dependence in Russia over this period. One can expect the correlation between monetary and other dimensions of well-being such as health and education to be much smaller under the Russian communist regime, given its reasonably well-funded universal educational and health care system. During the transition, the public funding of the educational and health care system decreased sharply, leading to a spontaneous commercialization and increased dependence between monetary and non-monetary dimensions of well-being. ${ }^{16}$ The RLMS is a rich panel data set so that the dependence between the dimensions of well-being of the same individuals can be analyzed over a relatively long period, which makes it a particularly interesting data set to test this hypothesis.

Over the last decade, the Russian Federation underwent a far-reaching transition from a centrally planned to a free market economy. Moreover, the country was hit by a severe financial crisis in August 1998. The transition and financial crisis had a large impact on many dimensions of everyday life in Russia. Many of these separate effects are by now well-documented in the literature. Real GDP per capita almost halved between 1990 and 1998, reaching in 2003 about $80 \%$ of its 1990 level. Russian mortality increased after 1990, with life expectancy falling from 64 years in 1990 to 58 years among men by 2002 and from 74 years to 72 years among women (World Development Indicators, 2008).

\footnotetext{
${ }^{16}$ See Blam and Kovalev (2006), Besstremyannaya (2007), Lokshin and Ravallion (2008) on the commercialization of the Russian health care sector and Smolentseva (2007) on the schooling system.
} 


\begin{tabular}{l|ll}
\hline & primary ranking variable & secondary ranking variable \\
\hline \hline standard of living & household income & individual income \\
health & self-assessed health & health indicator \\
schooling & years of schooling & additional courses \\
\hline
\end{tabular}

Table 1: Ranking Variables for the three dimensions of well-being.

Figure 2 presents the evolution of the Russian Human Development Index (HDI), a composite index combining average Russian performance on three dimensions of wellbeing: standard of living, life expectancy and education, with the latter captured by the literacy and the school enrolment ratio. The dark shaded bars of the figure show the analyzed period 1995-2003. After the steep decline in the early 90s, the HDI rises until the financial crisis strikes in 1998. After the financial crisis the HDI stagnates until its mild recovery in 2001. As explained in the introduction, the HDI is based on dimensionwise summary statistics and, hence, it is by construction insensitive to the dependence between its dimensions. To test if this is a mere academic concern, I look at the actual evolution of the dependence between three dimensions of Russian well-being: standard of living, health and schooling.

In the copula-based framework it suffices to know how the individuals are ranked in the different dimensions without having to know the exact outcome levels of the individuals, hence, ordinal ranking variables can be used. In all dimensions, individuals are ranked according to two ranking variables in a lexicographic way. In case of a tie in the primary ranking variable, the individuals are ranked according to the secondary ranking variable. If a tie occurs in all ranking variables for some individuals, they are ranked randomly. ${ }^{17}$ Table 1 surveys the ranking variables for the three dimensions of well-being. First, to obtain a ranking in the standard of living dimension, the individuals are ordered with respect to their equivalent real household incomes. ${ }^{18}$ In the case of ties, the individual real income over the last 30 days is used as secondary ranking variable. In this way, income pooling is assumed at the household level, but within each household the individuals who earn more are still ranked higher. The second dimension is the health dimension. The RLMS contains rich information on the health status of each respondent. Amongst other questions, the respondents are asked to assess their own health on a five-point scale. Selfassessed health has shown to be a very good predictor for overall health status or longevity in other surveys (for a comparison with other health indicators, see van Doorslaer and

\footnotetext{
17 See Denuit (2005) who extensively treats a similar procedure to make a continuous extension of a discrete random variable.

${ }^{18}$ Real household incomes are made comparable across different household sizes by applying the square root of the number of people in the household as an equivalence scale.
} 
Jones (2003)). Almost $60 \%$ of the individuals report to be of average health, therefore I use an objective health indicator as secondary ranking variable. The health indicator is constructed as a sum of six binary variables, each taking one if the individual reports that year to suffer from diabetes, a heart attack, anaemia, an operation, a hospitalization or other serious health problems. The ranking with respect to the third dimension, schooling, is obtained by the variable years of schooling. The individuals who spend the same amount of years in school are ranked based on the number of years they have followed any additional course, ranging from professional courses to music courses in art schools.

From the roughly 7000 individuals in the RLMS, a subsample of 1577 individuals in 1053 households is retained for whom I have complete information for all ranking variables in all waves of the survey. Although the full sample is constructed to be representative for the entire Russian Federation, this restricted balanced subsample might not be. Indeed, the individuals who remain in the sample in all waves are a bit poorer, less healthy and slightly less educated than the total sample. Working on the balanced subsample has the advantage that the same individuals are followed through time, so that changes in observed dependence reflect real changes in dependence and cannot be due to differences between the individuals who drop from the sample and the ones that are chosen to replace them.

For every pair of waves, it will be checked whether they can be ordered by the partial dependence relations introduced in section three. To see whether the sample of one wave is more dependent with respect to the lower orthant dependence relation than the one of another wave, one can apply definition 2 and check whether the empirical copula function in the first wave is everywhere not below the empirical copula function in the second wave, or equivalently whether the difference between all copula ordinates is nonnegative. Similarly, one uses definition 3 to test dependence with respect to the upper orthant dependence relation by looking at the difference between the ordinates of the joint survival functions. In theory, this procedure involves an infinity of tests (for each $p$ in $[0,1]^{m}$, the difference between all copula and joint survival function ordinates should be non-negative). Practically, however, the sign of the difference is only tested on a finite grid of position vectors $p$. For this three-dimensional illustration, a grid of 64 points is selected: $\{0.2,0.4,0.6,0.8\}^{3}$.

Since in each wave only a small sample from the underlying total Russian population is considered, it is interesting to know whether the results obtained actually hold for the total population as well; in other words, whether the obtained differences at every grid point are significantly non-negative. To test for significance, an approximation 


\begin{tabular}{l|lllllll}
\hline & 1995 & 1996 & 1998 & 2000 & 2001 & 2002 & 2003 \\
\hline \hline 1995 & - & 0 & 0 & 0 & 0 & 0 & 0 \\
1996 & 0 & - & 0 & 0 & 0 & 0 & 0 \\
1998 & 0 & 0 & - & 0 & 0 & 0 & 0 \\
2000 & 0 & 0 & 0 & - & 0 & 0 & 0 \\
2001 & 0 & 0 & 0 & 0 & - & 0 & 0 \\
2002 & 0 & 0 & 0 & 0 & L & - & 0 \\
2003 & L,U & $\mathrm{U}$ & 0 & 0 & L,U & 0 & - \\
\hline
\end{tabular}

Table 2: Pairwise partial dependence orderings (Source: author's calculations based on data from the RLMS, 1995-2003).

of the variance of the ordinates of the empirical copula and joint survival function is obtained by bootstrapping: from the original sample 1577 individuals are drawn with replacement repeatedly (1000 times). For each of the 1000 samples, I keep track of the obtained ordinates at all grid points, and the variance of these ordinates is used to test the significance of their difference. A conservative procedure is to reject the hypothesis of increased dependence if the differences between the copula or joint survival function ordinates of at least one grid point $p$ are significantly smaller than zero. (See also Dardanoni and Lambert (2001) and Cebrian, Denuit and Scaillet (2004) for a detailed treatment of different testing procedures for the concordance dependence relation). Table 2 summarizes all pair wise tests. Every cell presents the result of a test whether the row year exhibits higher dependence than the column year at the $5 \%$ significance level. An ' $L$ ' denotes lower orthant dependence, a ' $U$ ' denotes upper orthant dependence and a ' 0 ' denotes indecisiveness. If both dependence relations apply, the row year is more dependent according to the concordance dependence relation than the column-year. ${ }^{19}$

The dimensions of well-being are more dependent in 2003 than they were in 1995 and in 2001 according to the concordance dependence relation tested at the grid points $\{0.2,0.4,0.6,0.8\}^{3}$. It is a common finding in (one-dimensional) tests of stochastic dominance based on a finite number of grid points that the results are sensitive to the number of grid points chosen. It is not different here: for a finer grid none of the pairs can be ranked.

The grid can be chosen to focus on one of the tails of the empirical copula. Table 3 focusses on the lower tail of the empirical copula by considering the grid $\{0.1,0.2,0.3,0.4\}^{3}$ and Table 4 focusses on $\{0.6,0.7,0.8,0.9\}^{3}$. The lower tail of the distribution shows a

\footnotetext{
19 I experimented with many alternative ranking variables and the results are remarkably robust (results are available upon simple request).
} 
stronger increase in dependence than the upper tail, suggesting that especially the individuals at the bottom see their poor performance aggravated across dimensions. Note that the comparison in Table 4 between 2000 and 2001 with its focus on the upper tail shows a decrease in dependence.

\begin{tabular}{l|lllllll}
\hline & 1995 & 1996 & 1998 & 2000 & 2001 & 2002 & 2003 \\
\hline \hline 1995 & - & 0 & 0 & 0 & 0 & 0 & 0 \\
1996 & 0 & - & 0 & 0 & 0 & 0 & 0 \\
1998 & 0 & 0 & - & 0 & 0 & 0 & 0 \\
2000 & 0 & 0 & 0 & - & 0 & 0 & 0 \\
2001 & 0 & 0 & 0 & 0 & - & 0 & 0 \\
2002 & $\mathrm{U}$ & 0 & $\mathrm{U}$ & $\mathrm{U}$ & $\mathrm{U}$ & - & 0 \\
2003 & $\mathrm{U}$ & 0 & $\mathrm{~L}, \mathrm{U}$ & $\mathrm{U}$ & $\mathrm{L}, \mathrm{U}$ & 0 & - \\
\hline
\end{tabular}

Table 3: Pairwise partial dependence orderings with focus on the lower tail (Source: author's calculations based on data from the RLMS, 1995-2003).

\begin{tabular}{l|lllllll}
\hline & 1995 & 1996 & 1998 & 2000 & 2001 & 2002 & 2003 \\
\hline \hline 1995 & - & 0 & 0 & 0 & 0 & 0 & 0 \\
1996 & U & - & 0 & 0 & 0 & 0 & 0 \\
1998 & L & 0 & - & 0 & 0 & 0 & 0 \\
2000 & L & 0 & 0 & - & L & 0 & 0 \\
2001 & 0 & 0 & 0 & 0 & - & 0 & 0 \\
2002 & L & 0 & 0 & 0 & L & - & 0 \\
2003 & L & 0 & 0 & 0 & 0 & 0 & - \\
\hline
\end{tabular}

Table 4: Pairwise partial dependence orderings with focus on the upper tail (Source: author's calculations based on data from the RLMS, 1995-2003).

The large number of comparisons that are indecisive (especially on finer grids) is somewhat unfortunate. Therefore, in a next step, I look at the evolution of the dependence index $\widetilde{D}_{\perp}(X)$. Table 5 presents the results. The random ranking of individuals in case of double ties introduces some degree of arbitrariness in the results obtained. Therefore, I carry out a Monte Carlo experiment and repeat the calculation of the indices 1000 times. In every repetition the random assignment of the ranks in the case of double ties is done independently. The mean of these 1000 estimations is reported in the first column of Table 5. The second column of Table 5 provides the interval that includes $95 \%$ of the computed dependence indices. This interval is typically rather narrow, suggesting that the effect of random ranking in the case of ties is relatively small. Over the investigated 
decade, the dependence clearly increases.

One might be concerned how representative the results are for the total population. Therefore a second bootstrap is carried out. More specifically, I randomly draw 1000 times 1577 observations from the initial sample with replacement and compute the respective dependence indices. The mean of the 1577 indices obtained is almost identical to the indices reported in the first column of Table 5 and therefore not reported. The third column provides the intervals including $95 \%$ of the bootstrapped indices.

\begin{tabular}{cc|cc}
\hline year & index & $\begin{array}{c}95 \% \text { conf. interval } \\
\text { (Monte Carlo) }\end{array}$ & $\begin{array}{c}95 \% \text { conf. interval } \\
\text { (bootstrap) }\end{array}$ \\
\hline \hline 1995 & 0.136 & {$[0.128 ; 0.143]$} & {$[0.105 ; 0.170]$} \\
1996 & 0.151 & {$[0.144 ; 0.159]$} & {$[0.121 ; 0.183]$} \\
1998 & 0.143 & {$[0.135 ; 0.151]$} & {$[0.108 ; 0.176]$} \\
2000 & 0.164 & {$[0.155 ; 0.173]$} & {$[0.134 ; 0.196]$} \\
2001 & 0.141 & {$[0.132 ; 0.150]$} & {$[0.108 ; 0.172]$} \\
2002 & 0.187 & {$[0.179 ; 0.196]$} & {$[0.157 ; 0.223]$} \\
2003 & 0.210 & {$[0.200 ; 0.218]$} & {$[0.175 ; 0.240]$} \\
\hline
\end{tabular}

Table 5: Evolution of the dependence in Russia between 1995-2003, measured by multidimensional Spearman rank correlation coeffiicent $D_{\perp}(X)$ (Source: author's calculations based on data from the RLMS, 1995-2003).

Figure 3 depicts the evolution of the index. The full line shows the evolution of the index itself and the dashed and dotted lines present the $95 \%$ confidence intervals obtained by Monte Carlo and bootstrapping, respectively. The results show a clear and significantly increasing trend in the dependence during the decade after the collapse of the Soviet Union. Note that the results obtained from the dependence indices are indeed consistent with the concordance partial dependence relation of Table 2 as required theoretically. More detailed investigations are needed to understand better the underlying mechanisms why this is the case.

Finally, these findings provide a warning about the use of popular well-being measures based on summary statistics in every dimension, such as the HDI. The dependence between the dimensions of well-being in changing societies such as contemporary Russia is far from constant. Compare for instance the years 2002 and 2003 in Figure 2 and in Figure 3. It is clear that the Russian HDI increases, and that at the same time dependence increases. The standard of living, longevity and schooling on average improve, but more and more, the same individuals occupy the bottom and top positions in these 


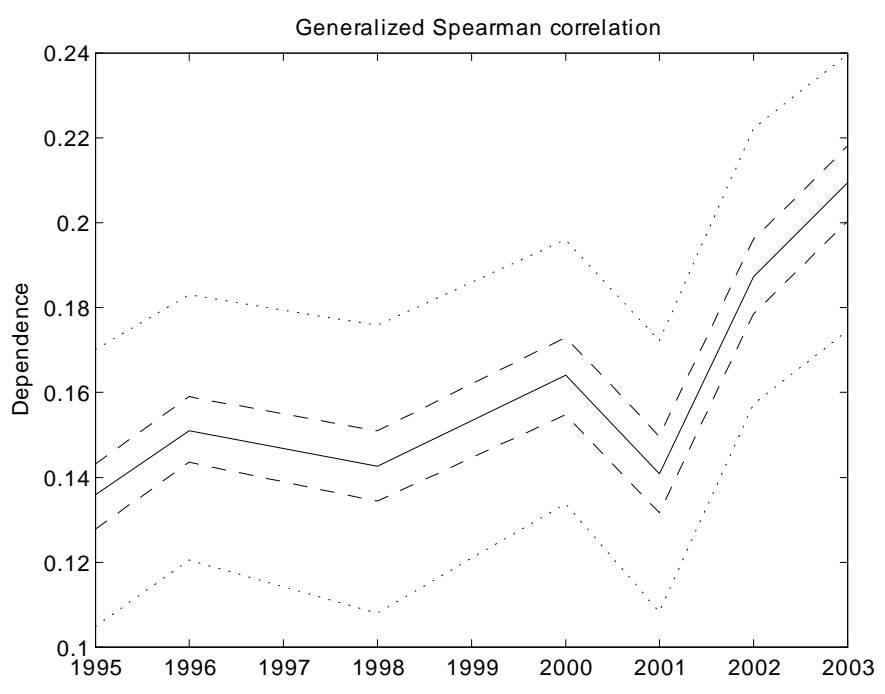

Figure 3: Dependence between the dimensions of well-being measured by the multidimensional Spearman rank correlation coefficient $\rho$ (Source: author's calculations based on data from the RLMS, 1995-2003).

dimensions. Furthermore, the increase in dependence is affecting mostly the lower tail of the well-being distribution.

\section{Conclusion}

This essay proposes a copula-based framework to analyze and quantify dependence between the many dimensions of well-being. The copula function allows one to formalize many of the theoretical notions from the literature on complex inequality in a natural way.

The copula-based partial concordance dependence relation compares the instances of outranking between individuals of two societies, thereby formalizing Miller's (1995) observation that in a more complex unequal society there are more cases of outranking between the individuals. The maximal element of the concordance dependence relation is the comonotonic society, in which one individual is top ranked in all dimensions, a second individual second ranked and so on. This society has been referred to in Walzer's (1983) original work as the maximally complex unequal society.

To quantify the evolution of complex inequality or dependence based on real-world data, it is convenient to impose more structure so that a complete ordering of all possible societies with respect to their dependence can be achieved. The present essay offers a 
simple axiomatic framework that leads to a class of copula-based dependence measures that is consistent with the partial concordance dependence relation, thereby addressing Dardanoni and Lambert's (2001) open question. Interestingly, a recently proposed multidimensional measure of dependence in the statistical literature, the multidimensional Spearman's rank correlation coefficient turns out to be a prominent member of the resulting class of measures.

The copula-based complete and partial dependence relations are applied to Russian data to investigate the claim that the correlation between dimensions of well-being increased during the Russian transition period. The analysis of the dependence in the RLMS allows one to shed some light on this claim. It is found that indeed the correlation showed a significant increase after the Russian transition to a free market economy. It is a question for further research whether this increase in dependence can also be found for other (transition) countries. However, the increased Russian dependence calls for a more careful use of composite multidimensional well-being measures based on dimension wise summary statistics, such as the HDI, which are by definition insensitive to dependence between the dimensions.

\section{References}

Arneson, R. (1995): “Against 'complex' equality," in Pluralism, Justice and Equality, ed. by D. Miller, and M. Walzer, pp. 226-252. Oxford University Press, Oxford.

Atkinson, A. B., And F. Bourguignon (1982): "The comparison of multidimensioned distributions of economic status," The Review of Economic Studies, 49(2), 183-201.

Behboodian, J., A. Dolati, and M. Úbeda-Flores (2007): "A multivariate version of Gini's rank association coefficient," Statistical Papers, 48(2), 295-304.

Besstremyannaya, G. (2007): "Out-of-Pocket health care expenditures by Russian consumers with different health status," Transition Studies Review, 14(2), 331-338.

Blam, I., and S. Kovalev (2006): "Spontaneous commercialisation, inequality and the contradictions of compulsory medical insurance in transitional Russia," Journal of International Development, 18(3), 407-423.

Bonhomme, S., and J.-M. Robin (2006): "Modelling individual earnings trajectories using copulas: France 1990 - 2002," in Contributions to Economic Analysis, Vol. 275: 
Structural Models of Wage and Employment Dynamics, ed. by H. Bunzel, B. Christensen, G. Neumann, and J.-M. Robin, p. chapter 18. Elsevier, Amsterdam.

Cebrian, A. C., M. Denuit, and O. Scaillet (2004): "Testing for concordance ordering," Astin Bulletin, 34(1), 151-173.

Colangelo, A., M. Scarsini, and M. Shaked (2006): "Some positive dependence stochastic orders," Journal of Multivariate Analysis, 97(1), 46-78.

DARDAnOni, V. (1996): "On multidimensional inequality measurement," in Research on Economic Inequality, ed. by C. Dagum, and A. Lemmi, vol. 6, pp. 201-205. JAI Press, London.

Dardanoni, V., and P. Lambert (2001): "Horizontal inequity comparisons," Social Choice and Welfare, 18, 799-816.

DecancQ, K. (2009): "How to order multidimensional distribution functions with respect to their dependence?," Mimeo.

Denuit, M., and P. Lambert (2005): "Constraints on concordance measures in bivariate discrete data," Journal of Multivariate Analysis, 93(1), 40 - 57.

Dolati, A., and M. Úbeda-Flores (2006): "On measures of multivariate concordance," Journal of Probability and Statistical Science, 4(2), 147-163.

Dutta, I., P. K. Pattanaik, and Y. Xu (2003): "On measuring deprivation and the standard of living in a multidimensional framework on the basis of aggregate data," Economica, 70(278), 197-221.

Embrechts, P., A. McNeil, and D. Straumann (2000): "Correlation and dependency in risk management: properties and pitfalls," in Risk Management: Value at risk and Beyond, ed. by M. Dempster, and H. Mofatt. Cambridge University Press, Cambridge.

Epstein, L. G., And S. M. TAnny (1980): "Increasing generalized correlation: a definition and some economic consequences," The Canadian Journal of Economics / Revue canadienne d'Economique, 13(1), 16-34.

Fleurbaey, M., and A. Trannoy (2003): "The impossibility of a Paretian egalitarian," Social Choice and Welfare, 21(2), 243-263.

Fournier, M. (2001): "Inequality decomposition by factor components: a "rankcorrelation" approach illustrated on the Tainwanese case," Recherches Economiques de Louvain, 67(4), 381-403. 
Haddar, J., and W. Russel (1974): "Stochastic dominance in choice under uncertainty," in Essays on Economic Behavior under Uncertainty, ed. by M. Balch, D. McFadden, and S. Wu, chap. 5, pp. 133-156. North-Holland, Amsterdam.

Hooghe, M. (1999): "The notion of complex equality and the beauty of Alcibiades," Ethical Perspectives, 6(3-4), 211-214.

Joe, H. (1990): "Multivariate concordance," Journal of Multivariate Analysis, 35, 12 30 .

(1997): Multivariate Models and Dependence Concepts. Monographs on Statistics and Applied Probability no. 73. Chapmann and Hall, London.

Lokshin, M., and M. Ravallion (2008): "Testing for an economic gradient in health status using subjective data," Health Economics, 17(11), 1237-1259.

MaAsoumi, E. (1999): "Multidimensioned approaches to welfare analysis," in Handbook of Income Inequality Measurement, ed. by J. Silber, pp. 437-477. Kluwer Academic, Boston.

Miller, D. (1995): "Complex equality," in Pluralism, Justice and Equality, ed. by D. Miller, and M. Walzer, pp. 197-225. Oxford University Press, Oxford.

Moulin, H., And W. Thomson (1997): "Axiomatic analyses of resource allocation problems," in Social Choice Re-examined, ed. by K. J. Arrow, A. K. Sen, and K. Suzumura, pp. 101-120. St. Martin's Press, New York.

Nelsen, R. B. (1991): "Copulas and association," in Advances in Probability Distributions with Given Marginals and Statistical Modelling, ed. by G. Dall'Aglio, S. Kotz, and G. Salinetti. Kluwer, Dordrecht.

(2002): "Concordance and copulas: a survey," in Distributions with Given Marginals and Statistical Modelling, ed. by G. Dall'Aglio, S. Kotz, and G. Salinetti, pp. 169-178. Kluwer, Dordrecht.

(2006): Introduction to Copulas. Springer Series in Statistics no. 139. Springer Verlag, New York.

Pogge, T. (2002): "Can the capability approach be justified?," Philosophical Topics, $30(2), 167-228$.

Quinn, C. (2007): "Using copulas to measure association between ordinal measures of health and income," Health, Econometrics and Data Group (HEDG) Working Papers $07 / 24$. 
(2009): "Measuring income-related inequalities in health using a parametric dependence function," Health, Econometrics and Data Group (HEDG) Working Papers $09 / 24$.

SCARSini, M. (1984): "On measures of concordance," Stochastica, 8, 201-218.

Schmid, F., And R. Schmidt (2006): "Bootstrapping spearman's multivariate rho," in COMPSTAT 2006 Proceedings in Computational Statistics, ed. by A. Rizzi, and M. Vichi, pp. 759-767. Physica-Verlag, Oporto, Portugal.

(2007): "Multivariate conditional versions of Spearman's rho and related measures of tail dependence," Journal of Multivariate Analysis, 98(6), 1123-1140.

Schweizer, B., And A. Sklar (1983): Probabilistic Metric Spaces. North Holland, New York.

Schweizer, B., And E. F. Wolff (1981): "On nonparametric measures of dependence for random variables," The Annals of Statistics, 9(4), 879-885.

Sklar, A. (1959): "Fonctions de répartition à $n$ dimensions et leurs marges," Publications de l'Institut de Statistique de L'Université de Paris, 8, 229-231.

Smolentseva, A. (2007): "Educational inequality in Russia," in International Studies in Educational Inequality, Theory and Policy Volume 2: Inequality in Education Systems, ed. by R. Teese, S. Lamb, and M. Duru-Bellat, pp. 143-156. Springer, Netherlands.

Swift, A. (1995): "The sociology of complex equality," in Pluralism, Justice and Equality, ed. by D. Miller, and M. Walzer, pp. 253-280. Oxford University Press, Oxford.

TAYlor, M. (2007): "Multivariate measures of concordance," Annals of the Institute of Statistical Mathematics, 59(4), 789-806.

van Doorslaer, E., And A. Jones (2003): "Inequalities in self-reported health: validation of a new approach to measurement," Journal of Health Economics, 22(1), $61-87$.

Walzer, M. (1983): Spheres of Justice: A Defense of Pluralism and Equality. Basic Books, New York.

WEYMARK, J. A. (2006): "The normative approach to the measurement of multidimensional inequality," in Inequality and Economic Integration, ed. by F. Farina, and E. Savaglio. Routledge, London. 


\section{Appendix: Proof}

Proposition. If the binary relation $\succsim^{*}$ on the set of random vectors satisfies ORD, CONT, IND and CONC, and if the real-valued function $S$ has non-negative even crossderivatives, then $Y \succsim^{*} X$ implies that:

$$
D(Y)=\int_{[0,1]^{m}} S(p) d C_{Y}(p) \geq \int_{[0,1]^{m}} S(p) d C_{X}(p)=D(X) .
$$

Proof. Step 1. Fishburn (1970, Theorem 8.2) shows that a binary relation ${ }^{*}$ on the set of random vectors satisfying ORD, CONT and IND can be represented by a function $D$ of the form

$$
D(X)=\int_{\mathbb{R}^{m}} S(x) d F_{X}(x),
$$

with $S: \mathbb{R}^{m} \rightarrow \mathbb{R}$ a real-valued function.

Step 2. Consider the random vector $P$ which is obtained by transforming the random variables of $X$ by their respective marginal distribution function. Since the marginal distribution functions are all increasing and continuous, Theorem 2 (Schweizer and Wolff, 1981) can be evoked to show that $X$ and $\Phi(X)$ have the same copula function and joint survival function. Hence, by the axiom CONC, $D(X)=D(P)$ so that:

$$
D(X)=\int_{[0,1]^{m}} S(p) d C_{X}(p),
$$

with $S: I^{m} \rightarrow \mathbb{R}$ a real-valued function.

Step 3. Copula functions are joint distribution functions, so by Theorem 5.8 from Hadar and Russel (1974) $C_{X}(p) \geq C_{Y}(p)$ implies:

$$
\int_{[0,1]^{m}} S(p) d C_{X}(p) \geq \int_{[0,1]^{m}} S(p) d C_{Y}(p)
$$

for all real-valued functions $S$ with positive even cross derivatives and negative odd cross derivatives. By a similar argument it holds that $\bar{C}_{X}(p) \geq \bar{C}_{Y}(p)$ implies:

$$
\int_{[0,1]^{m}} S(p) d C_{X}(p) \geq \int_{[0,1]^{m}} S(p) d C_{Y}(p)
$$

for all real-valued functions $S$ with positive cross derivatives. Combining (7) and (8) leads to the result that $Y \succsim_{C} X$ implies that:

$$
\int_{[0,1]^{m}} S(p) d C_{Y}(p) \geq \int_{[0,1]^{m}} S(p) d C_{X}(p),
$$

for all the real-valued function $S$ with non-negative even cross-derivatives. 